Check for updates

Cite this: J. Mater. Chem. A, 2020, 8 , 4898

Received 4th November 2019

Accepted 16th February 2020

DOI: $10.1039 / \mathrm{c} 9 \operatorname{ta} 12127 \mathrm{~g}$

rsc.li/materials-a

\section{Femtosecond laser-induced surface structuring of the porous transport layers in proton exchange membrane water electrolysis $\uparrow$}

\author{
Michel Suermann, (D) $\ddagger^{\star a}$ Thomas Gimpel, (D) $\ddagger^{\mathrm{b}}$ Lena V. Bühre, (D) ${ }^{a}$ Wolfgang Schade, ${ }^{\mathrm{bc}}$ \\ Boris Bensmann (D) and Richard Hanke-Rauschenbach ${ }^{a}$
}

In proton exchange membrane water electrolysis (PEMWE) cells the performance and thus the conversion efficiency are influenced by the interface between the porous transport layer (PTL) and the catalyst layer $(\mathrm{CL})$. In the following paper, this interface is modified by the use of femtosecond laser-induced surface structuring, so that the specific surface area of the titanium based fibers of the PTL is increased. The resulting morphology exhibits two roughness levels of (i) a relatively coarse structure featuring tips of a few micrometers in diameter and depth, which are each covered in turn by (ii) a substructure of smaller tips of a few to several hundred nanometers in diameter and depth. PEMWE electrochemical characterization and short-term stress tests reveal that the cell performance is increased due to the laser-structuring of the PTL surface towards the $\mathrm{CL}$. For instance, the cell voltage is reduced by approximately $30 \mathrm{mV}$ after $100 \mathrm{~h}$ at $4 \mathrm{~A} \mathrm{~cm}^{-2}$. These beneficial effects are observed over the entire current density range and thus correspond to a decreased equivalent cell resistance of at least $6 \mathrm{~m} \Omega$ $\mathrm{cm}^{2}$ for electrical interfacial contact losses and at least $2 \mathrm{~m} \Omega \mathrm{cm}^{2}$ for mass transport losses. A physical characterization by scanning electron microscopy shows that the $\mathrm{CL}$ surface is much rougher and more jagged when using laser-structured fibers. Thus, the gaseous oxygen and the liquid water transport both from and to the active sites of the catalyst seem to be improved.

\section{Introduction}

Efficient energy storage solutions are becoming increasingly important to meet a growing energy demand with a rising share of renewable energy sources. One reason is the naturally intermittent electricity production of wind and solar power plants. ${ }^{1}$ Power to gas plants are favored for energy storage over a longer period of days to months as well as for large quantities. For these applications, proton exchange membrane water electrolysis (PEMWE) is considered a promising technology. ${ }^{2}$ There are already plants in the MW range, ${ }^{3}$ which are expected to increase to a scale of several hundred MW or even GW in the near future.

Nevertheless, there are still a number of development gaps to further reduce the capital expenditure (CAPEX) and operational expenditure (OPEX)., ${ }^{\mathbf{4} 5}$ A possibility to further decrease the OPEX is to increase the efficiency of the electrolyzer by e.g.

\footnotetext{
${ }^{a}$ Institute of Electric Power Systems, Leibniz Universität Hannover, 30167 Hannover, Germany. E-mail: suermann@ifes.uni-hannover.de

${ }^{b}$ Clausthal University of Technology, Research Center Energy Storage Technologies, 38640 Goslar, Germany

'Fraunhofer Heinrich Hertz Institute, 38640 Goslar, Germany

$\dagger$ Electronic supplementary information (ESI) available. See DOI: $10.1039 /$ c9ta12127g

\$ These authors contributed equally to this work.
}

reducing the overpotentials at a given hydrogen output, respectively current density. The overpotentials are usually further distinguished in the three main types, i.e. kinetic or activation, ohmic and mass transport or concentration overpotential. The overpotential reduction can be achieved either by optimizing the operating conditions such as temperature and pressure and/or by optimizing the materials and components.

With regard to materials and components, particularly the anode interface between the porous transport layer (PTL) and the catalyst layer (CL) has gained in importance in recent years. While a carbon-based PTL can be used at the cathode, similar to the materials used in its sister technology PEM fuel cells (PEMFCs), the relatively harsh anodic conditions in PEMWE limit the choice mostly to titanium-based sintered powder, fiber or mesh type PTLs. Furthermore, these titanium-based PTLS were originally developed for the filter industry and are not optimized for the use in PEMWE cells, as reviewed in e.g. ref. 5 and 6. In other words, there are still a lot of possibilities for optimization.

Thus, mainly the bulk structure of the PTL and the corresponding pore and particle size distribution have been studied by several research groups (see e.g. Grigoriev et al., ${ }^{7}$ Ito et al., ${ }^{\mathbf{8}, \mathbf{9}}$ Hwang et al.,$^{\mathbf{1 0}}$ and Kang et al. $\left.{ }^{\mathbf{1 1 - 1 4}}\right)$ pointing out a significant influence towards the performance of the cells. Further attempts to correlate the bulk geometric and transport 
properties of the PTL with the individual overpotentials haven been made, as briefly summarized below. Suermann et al. ${ }^{15,16}$ and Bernt et al. ${ }^{17}$ found out that the mass transport overpotential decreases with increasing operating pressure, both for the anode and cathode side in symmetric and asymmetric electrochemical self-pressurized configurations. However, at anode pressures in the higher two-digit bar range the remaining mass transport overpotentials were practically independent of the PTL structure. Similar to $\mathrm{H}_{2} / \mathrm{N}_{2}$-experiments for PEMFC materials, ${ }^{18}$ Babic et al. measured influences of the PTL structure $^{19}$ and the catalyst loading ${ }^{20}$ to the proton transport resistance in the $\mathrm{CL}$, which is attributed to the mass transport overpotential (determined in this manner). The supposed breakthrough has been achieved by Schuler et al. ${ }^{21,22}$ who have found a correlation between the overpotentials to the interfacial contact area between the PTL fibers and the CL. It should be noted, that in their work all three main overpotentials have been influenced by the fiber diameter and/or the porosity of the PTL. This should only provide a small insight in the topic. In the further course of this paper, we will discuss the literature results relevant to this work in more detail.

In summary, not only the electrical interfacial contact resistances between the PTL and the CL are of interest, but also the influence of the PTL/CL-interface towards the kinetic and mass transport overpotential should be kept in mind. In this work, the interface of the PTL towards the CL is structured with a femtosecond laser to increase its specific surface area and thus contact area, in order to decrease the aforementioned electrical interfacial contact resistances in particular. The technique has been already used to improve the performance of alkaline electrolysis electrodes. ${ }^{23-25}$ To the best of our knowledge, it is the first time such an approach is used for titaniumbased PTLs in PEMWE. The laser-structuring process step is interesting both for a more fundamental understanding in the physicochemical and electrochemical processes at the PTL/CLinterface and for the industry to further optimize the components.

\section{Experimental}

\section{Electrochemical characterization}

The electrolysis cell tests are carried out on a commercial PEMWE test rig (Greenlight Innovation, E40). At the anode, the cell is supplied with a constant water recirculation flow of $20 \mathrm{ml}$ $\left(\min ^{-1} \mathrm{~cm}^{-2}\right)$. The water conductivity is kept below or equal to $0.1 \mu \mathrm{sm}^{-1}$ at all times due to an implemented ion exchanger with a mixed resin. At the cathode, no water is supplied. All electrolysis tests are performed at an operating temperature of $60{ }^{\circ} \mathrm{C}$ and at ambient pressure.

A commercial PEMWE cell setup with a geometric active area of $4 \mathrm{~cm}^{2}$ and a separate electric heating for the cell housing (balticFuelCells, quickCONNECT) is used. Considering the relatively high excess water and the small active area, it can be considered as a differential cell mimicking the inlet part of a technical cell. Within this cell type, the contact pressure on the active surface is set via a pneumatic actuator and independent of the contact pressure of the gasket. While the influence of the contact pressure is investigated in ex situ tests, the operando electrolysis cell tests are performed with a constant gas pressure of 2 bar(g). According to manufacturer's specifications, this corresponds to a contact pressure of about 38 $\operatorname{bar}(\mathrm{g})$.

For the operando tests commercially available state-of-theart Nafion 115 based catalyst coated membranes (CCMs) are used (Greenerity, E400). At the anode, titanium fiber based PTLS with a nominal thickness of $1 \mathrm{~mm}$, a porosity of $56 \%$ and a fiber diameter of $20 \mu \mathrm{m}$ (Bekaert, 2GDL40-1.00) are used. On the cathode side, a carbon-based PTL with a hydrophobic treatment is used, which is designed for electrolysis operation according to manufacturer's specifications (Freudenberg, H23l2). In addition, for the ex situ tests, similar additional Ti-PTLs are used which are similar to the aforementioned anodic Ti-PTLs except for their nominal thickness of $0.2 \mathrm{~mm}$.

Prior to the cell tests, before to the laser treatment and prior to the physical examinations, the PTLs are washed in DI-water in an ultrasonic bath for $15 \mathrm{~min}$ at room temperature. With respect to the operando electrolysis cell tests, the CCMs are assembled in a dry state, as received from the manufacturer. The cell is then rinsed with water heated to $60{ }^{\circ} \mathrm{C}$ for one hour to ensure a full humidification of the membrane. ${ }^{26}$

All experiments are performed with a potentiostat (Bio-Logic, SP-150 with 20 A booster). For operando electrolysis cell tests, a galvanostatic protocol, originally developed to systematically study the influence of specific parameters on degradation (details can be found in ref. 27), is slightly adapted. First the PEMWE cells undergo a conditioning phase consisting of three repetitions of polarization curves ( $i / E$-curves), electrochemical impedance spectroscopy (EIS) measurements and current interrupt (CI) measurements. The three repetitions of $i / E$ curves, EIS and CI measurements are hereinafter referred to as the characterization phase. The measurement protocols are listed separately below.

Afterwards, a constant current of $1 \mathrm{~A} \mathrm{~cm}^{-2}$ is applied for 10 hours serving as an internal benchmark to decide whether the cell performs as expected or not. Unexpected behavior may result from incorrect assembly or contamination of the components. During this work, this source of error could be completely avoided and all samples fulfilled the criteria resulting in an outstanding reproducibility. After completing the conditioning phase, the actual experiments begin. This stage is hereinafter referred to as the begin of tests (ВОT). Subsequently, an electrochemical characterization phase with three repetitions of $i / E$-curves, EIS and CI measurements is performed (again), followed by a constant current density phase at $4 \mathrm{~A} \mathrm{~cm}^{-2}$ lasting for 30 hours. The last two phases are repeated until the end of test (EOT), which is not chosen for reasons of actual degradation, but rather because of limited test rig capacity, and thus serve as a short-term stress test. To emphasize this, and as shown below, the degradation of all samples/cells is in the usual range. In addition, the focus of this work is on a proof of concept, therefore a long-term test unfortunately goes beyond the scope of this work, especially when considering lifetimes of PEMWE cells and systems are in the range of years rather than months or even days. 
Nevertheless, a short-term stress test at elevated current densities of $4 \mathrm{~A} \mathrm{~cm}^{-2}$ is provided up to 150 hours, depending on the cell and series of measurement.

The PEMWE cells are characterized with $i / E$-curves with logarithmic decimal current density steps. The holding time is 10 seconds plus another 22 seconds for a high frequency resistance (HFR) measurement from 100 to $0.1 \mathrm{kHz}$ at each current density step. While such short holding times are rather unusual for PEM fuel cells, due to the current density dependent membrane humidification, these are rather unproblematic in electrolysis conditions, as the membrane is always in contact with liquid water. ${ }^{15}$ The HFR is determined by linear interpolation at the intersection of the real part axis in the Nyquist plot, where the imaginary part is equal to zero.

The cells are further characterized with the help of EIS measurements at $0.01,0.1,0.2,0.3,0.4,0.6,0.8,1,2,3$ and $4 \mathrm{~A} \mathrm{~cm}^{-2}$ from $100 \mathrm{kHz}$ to $100 \mathrm{mHz}$ each. The alternating current is $10 \%$ of the superimposed direct current, as it is used for the aforementioned HFR measurements.

With regard to the electrical connection of the cell, the current cables are connected to the endplates and the sense cables to the flow field housing. Although qualitatively similar, an offset of about $7 \mathrm{~m} \Omega \mathrm{cm}^{2}$ is measured between these two points due to additional electrical interfacial contact resistances. In this work, all data shown are based on measurements at the flow field housing.

\section{Femtosecond laser structuring}

This work addresses the titanium-based PTLs. Their surface towards the CL is laser-structured using a Mantis seed laser from Coherent and a Spitfire $10 \mathrm{kHz}$ regenerative amplifier from Spectra Physics at a wavelength of $800 \mathrm{~nm}$ and a pulse length of about $60 \mathrm{fs}$. This work should be seen as proof of concept. In order to prepare the selected surface structure, the laser beam is focused through a $500 \mathrm{~mm}$ lens to a measured laser spot diameter of about $80 \mu \mathrm{m}$ (intensity 1/e2) into a process chamber under continuous $\mathrm{N}_{2}$ process gas flow at pressures of approximately 800 mbar. Area processing occurs by a meandering scan with a linear scan velocity of $2.8 \mathrm{~mm} \mathrm{~s}^{-1}$ corresponding to about 285 pulses per spot on the sample.
Furthermore, an appropriate overlap of adjacent lines is realized with a line pitch of $60 \mu \mathrm{m}$. The laser fluency is set to $3.0 \mathrm{~J} \mathrm{~cm}^{-2}$.

\section{Physical characterization}

The surface of the pristine (or unmodified) and laser-structured titanium-based PTLs towards the CL as well as the anode CL surface are examined by scanning electron microscopy measurements both before and after the electrochemical characterization (SEM, Zeiss EVO® MA 10). The SEM-images of the PTL surface and of the CCMs are taken at an angle of $45^{\circ}$ and from the top, respectively. For the post operando SEM-images, the Ti-PTL/CCM/C-PTL sandwiches are carefully disassembled from the electrolysis cells and separated. During the transport, each sandwich is stored in a humid, closed-air atmosphere in order to maintain the morphology of the components at the interface as good as possible.

\section{Results}

First, the SEM-results of the pristine (or unmodified) and laserstructured PTLs are shown in order to illustrate the morphological changes caused by the laser-treatment. Subsequently, the results of the ex situ and operando cell tests are given. Then, the SEM-images of the PTL and CCM surface after electrochemical characterization are presented in order to show the effects of the electrochemical testing procedure towards the morphology. Finally, all observed changes and effects are discussed in order to increase the fundamental understanding and to provide a meaningful basis for further work.

\section{Physical characterization before cell tests}

In Fig. 1 the SEM-images of the surface of the pristine (top) and laser-structured (bottom) PTLs are shown, which are in contact to the anode CL later. While the surface morphology of the pristine fibers is rather smooth, a relatively rough and jagged microstructure is observed after the laser treatment. In addition to a coarse structure characterized by tips of a few micrometers in diameter and depth, each tip is covered with a substructure

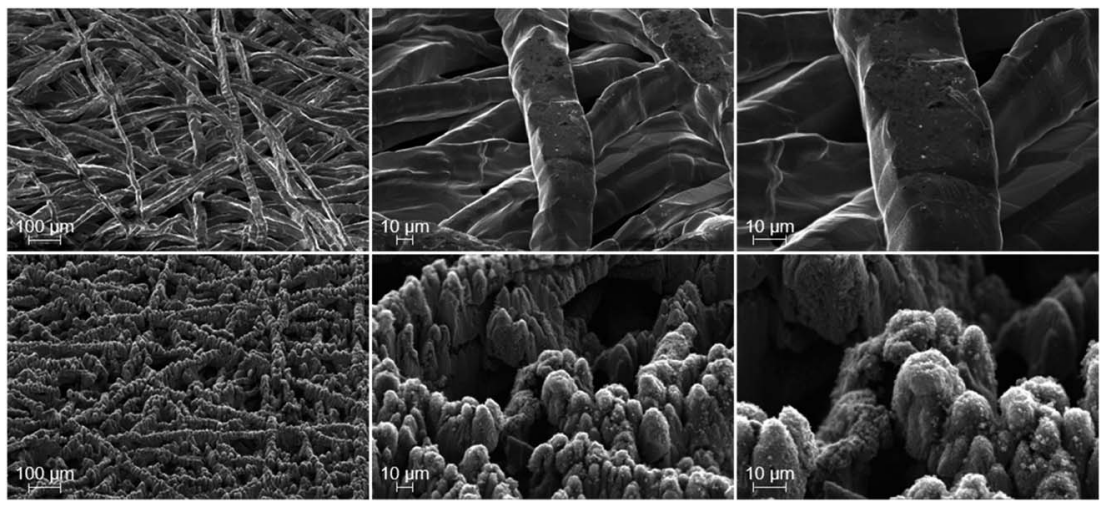

Fig. 1 SEM-images before PEMWE cell tests of the PTL surface, which is later in contact with the CL, of the pristine (top) and the laser-structured PTLs (bottom) each at magnifications of $100 \times, 500 \times$ and $1000 \times$, from left to right. 
of smaller (sintered) flakes with dimensions of a few to several hundred nanometers in diameter and depth. The substructures partially correspond to laser-induced periodic surface structures (LIPSS). ${ }^{28}$ Consequently, an increase in the roughness and thus in the specific surface area of the PTL fibers is achieved due to the laser-structuring.

\section{Ex situ electrical testing}

In order to provide an initial assessment, the electrical ohmic resistance of the pristine and laser-structured PTLs is measured in an ex situ cell setup, i.e. without a CCM. The design of the experiment is similar to those in ref. 17 and 29 and allows a HFR measurement to account for the sum of the electrical bulk and interfacial contact resistances. However, the latter is the dominating resistance due to the relatively high intrinsic electrical bulk conductivity of the materials used, which is in the order of magnitude of $5 \times 10^{5} \mathrm{~S} \mathrm{~m}^{-1}$ in through-plane direction for such fiber-based Ti-PTLs. ${ }^{21}$ In this work, three different setups are used. In each setup the PTL samples are in contact with a (i) a carbon-based PTL, (ii) a titanium-based PTL and (iii) a bare flow field, as depicted in Fig. 2.

As expected, higher contact pressures result in lower HFR, especially for the setup (i) vs. the compressible C-PTL. Furthermore, the results of the metal/metal-interfaces in setup (ii) and (iii) are qualitatively and quantitatively similar to a large extent. With respect to the laser-structuring, a reduction of the HFR of about $20-50 \%$ can be reported for all investigated setups and contact pressures. This improvement can be explained first and foremost by the reduced electrical interfacial contact resistances. Now, however, the question arises whether or not these improvements are transferable to operando electrolysis cell tests.

\section{Operando electrochemical testing}

In this section, data of the electrochemical tests are shown after completing the conditioning step, i.e. after approximately three hours of ramping up between 0 and $4 \mathrm{~A} \mathrm{~cm}^{-2}$ and a subsequent $10 \mathrm{~h}$ constant current period at $1 \mathrm{~A} \mathrm{~cm}^{-2}$. For details, please see ref. 27. Thus, the following shown data is at the time after completing the conditioning step, i.e. at the BOT.

Overpotential analysis at BOT. Current voltage characteristics in the form of polarization curves up to $4 \mathrm{~A} \mathrm{~cm}^{-2}$ are performed at $60{ }^{\circ} \mathrm{C}$ for the four cells, two with pristine Ti-PTLs, labelled as P1 and P2, and two with laser-structured Ti-PTLs, labelled as L1 and L2. Furthermore, the HFR is measured at each current step. As usual, the HFR accounts for the electric and ionic interfacial and bulk transport resistances and is obtained at about $3 \mathrm{kHz}$.

As illustrated in Fig. 3a, a significant and reproducible increase in cell performance is achieved by the laser-structuring of the PTL surface towards the CL. For instance, at $4 \mathrm{~A} \mathrm{~cm}^{-2}$ the cell voltage is reduced from $2.325 \pm 0.006 \mathrm{~V}$ (P1 and P2) to 2.267 $\pm 0.007 \mathrm{~V}$ (L1 and L2) by an impressive value of $58 \mathrm{mV}$. Reproducibility was demonstrated in each case by two identical series of measurements, both for experiments with laser-

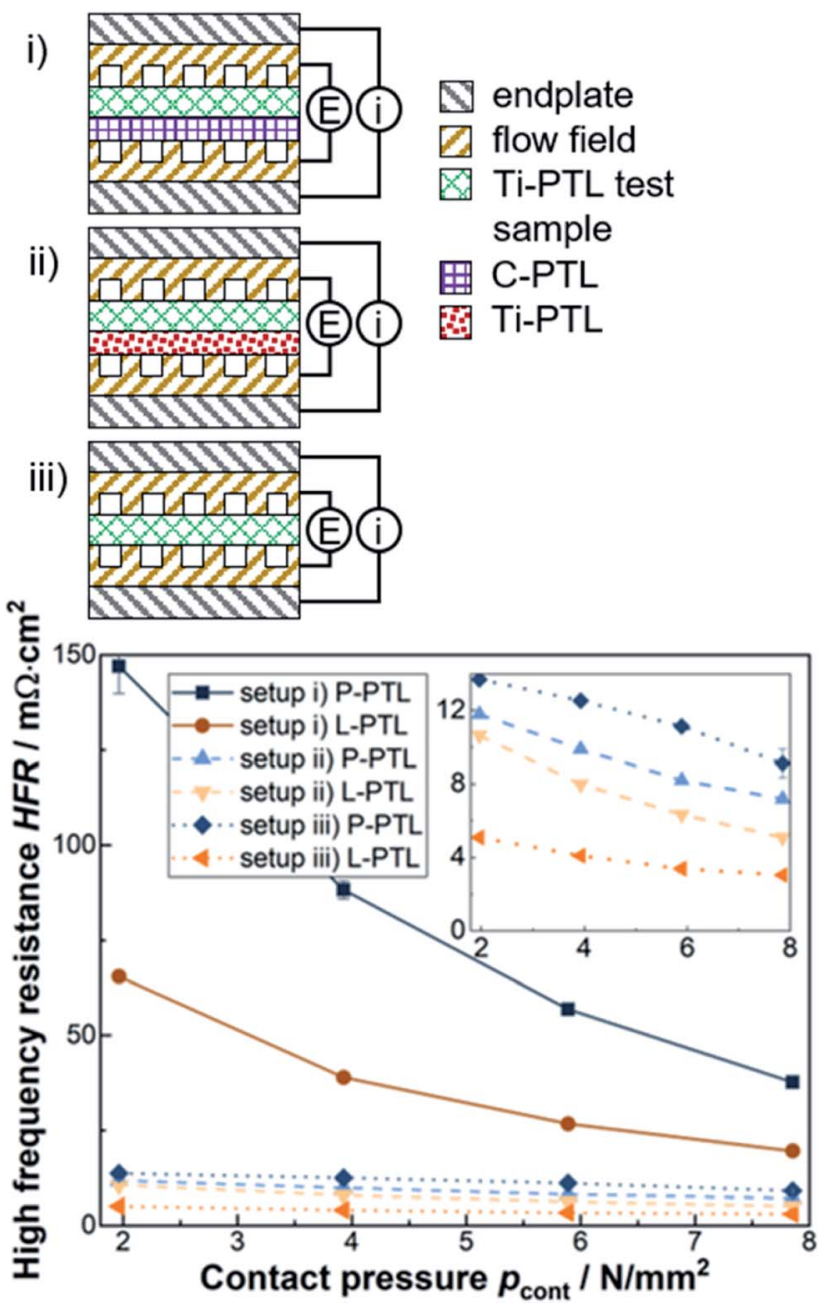

Fig. 2 Ex situ electrical tests with the laser-structured (L-)PTLs and with the pristine (P-)PTLs. (top) Schematic design of the three ex situ electrical tests with setup (i) vs. a carbon based PTL (solid lines in the bottom figure) (ii) vs. a thin titanium-based PTL (dashed lines) and (iii) vs. a bare titanium-based flow field (dotted lines). (bottom) Corresponding area-specific HFR measurements as a function of the applied contact pressure. $3.8 \mathrm{~N} \mathrm{~mm}^{-2}$ is used for subsequent electrolysis tests. The inset shows the results for the setups II and III in detail. The values shown correspond to the mean values with the corresponding standard deviations of 4 different measurements at $0.1,0.4,1$ and $4 \mathrm{~A} \mathrm{~cm}^{-2}$ (all at $40^{\circ} \mathrm{C}$ ). As expected, the HFR is not dependent on the current density

structured and pristine PTLs. These beneficial effects are observed over the entire current density range showing a pseudo-linear behavior. Thus, the corresponding equivalent area-specific cell resistance is reduced by about $14 \mathrm{~m} \Omega \mathrm{cm}^{2}$. What is the reason for this observation? As mentioned before, the original aim is to improve the electrical interfacial contact resistances between the PTL and the CL. In fact, this was achieved analogous to the aforementioned ex situ experiments due to a reduction of the HFR with increasing current density from 6 up to $9 \mathrm{~m} \Omega \mathrm{cm}^{2}$ (see Fig. 3a). Thus, the HFR improvement accounts for about two thirds of the total improvement.

Consequently, the missing one third has to be assigned to other overpotentials. When considering the differentiation of 

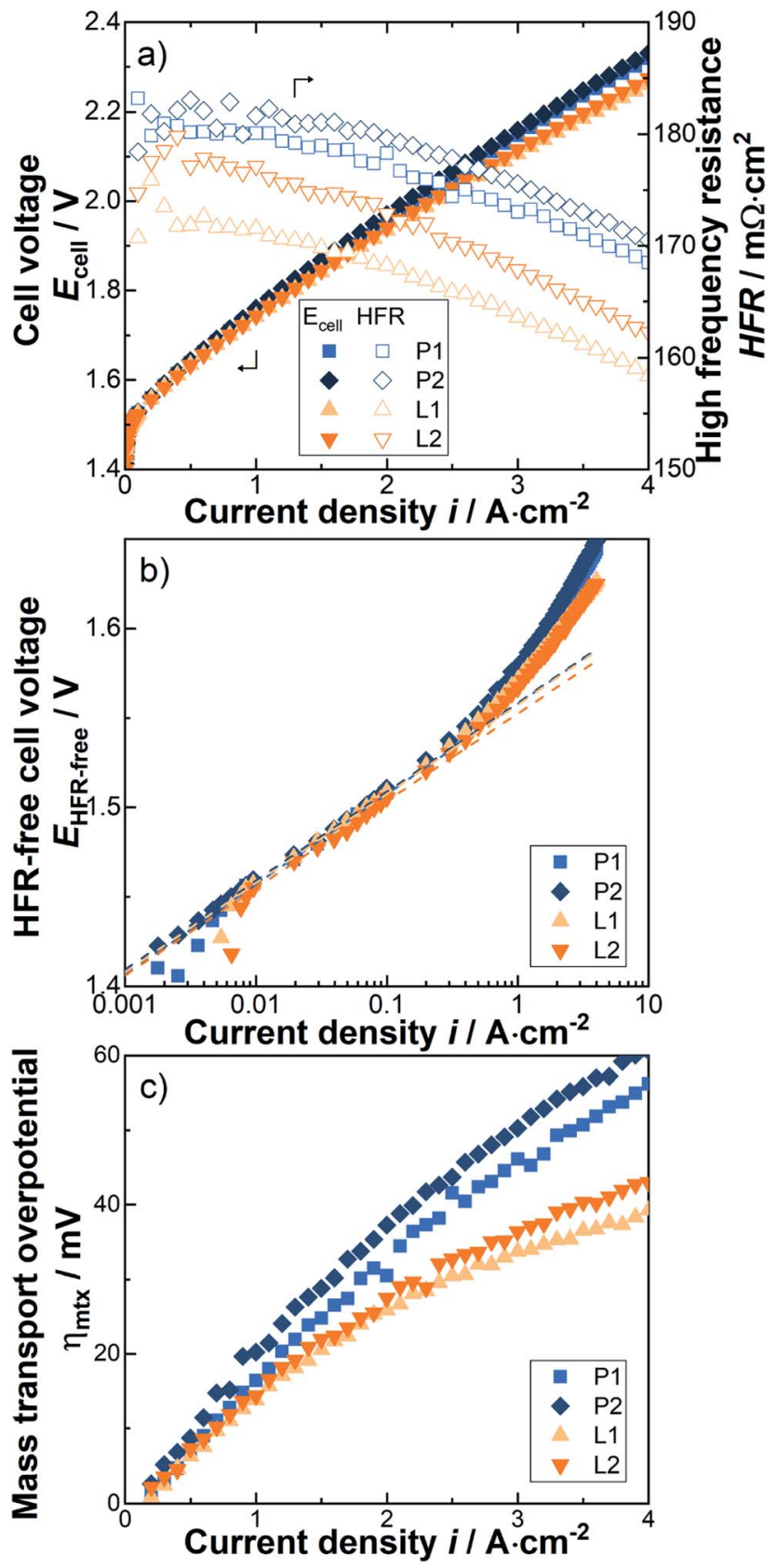

Fig. 3 (a) Polarization curves and area specific HFR. (b) Tafel-plot and corresponding (extrapolated) Tafel-lines. The Tafel-lines are fitted between 10 and $100 \mathrm{~mA} \mathrm{~cm}^{-2}$. (c) The difference between the HFRfree cell voltage and the extrapolated Tafel-lines, i.e. mass transport overpotentials.

the total overpotential into the three major types of overpotential, then either the kinetics or the mass transport must be improved along with the ohmic part. To further distinguish these two types of overpotentials, an analysis based on the Butler Volmer approach and transition state theory is applied..$^{30}$ Thus, the HFR-free cell voltage is plotted against the current density in a so called Tafel-plot and the Tafel-line is fitted in the linear region where the mass transport losses are assumed to be negligible, i.e. between 0.01 and $0.1 \mathrm{~A} \mathrm{~cm}^{-2}$. It should be noted that, by definition, the kinetics are then completely assigned to the rate-determining step within the oxygen evolution reaction due to its sluggish reaction kinetics compared to the faster and quasi-reversible hydrogen evolution reaction kinetics. ${ }^{31,32}$ For details of the applied overpotential breakdown method, please have a look at ref. 15.

With respect to the OER kinetics, it is difficult to determine any difference due to the laser-structuring, as the Tafel slope is

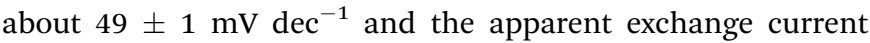
density is about $(4 \pm 1) \times 10^{-8} \mathrm{~A} \mathrm{~cm}^{-2}$ for all investigated cells. At most, there is a trend towards a negligibly small improvement, which lies within the error deviation, in the form of reduced OER overpotentials and Tafel-slopes due to laserstructuring (Fig. 3b). In order to be able to make a more precise statement, however, further series of measurements are required. Nevertheless, such an improvement is conceivable, as the results of Schuler et al..$^{22}$ have shown a dependency when significantly changing the PTL fiber diameter and porosity. Consequently, and somewhat surprisingly, the missing one third of the total improvements can be assigned to the mass transport overpotential.

The mass transport overpotential is determined by the difference between the HFR-free cell voltage and the extrapolated Tafel-line and is shown separately in Fig. 3c. For all cases, the corresponding equivalent mass transport resistances reduce with increasing current density, e.g. for the cells with pristine PTLs from about 18 to $15 \mathrm{~m} \Omega \mathrm{cm}^{2}$ and for the cells with laserstructured PTLs from about 14 to $10 \mathrm{~m} \Omega \mathrm{cm}^{2}$. Thus, a qualitatively similar curve progression of the resulting ohmic overpotential can be observed, which slightly changes from an almost linear to a more logarithmic increase with increasing current density. It should also be emphasized that the laserstructuring of the PTL surface towards the CL results in a significant reduction in the mass transport resistances of just over $4 \mathrm{~m} \Omega \mathrm{cm}^{2}$ over the entire current density range.

In summary, the laser-structuring of the PTL surface towards the CL increased the BOT cell performance, e.g. at $4 \mathrm{~A} \mathrm{~cm}^{-2}$ of about $58 \mathrm{mV}$. Throughout the entire current density range, the corresponding equivalent cell resistance is reduced by about 9 to $15 \mathrm{~m} \Omega \mathrm{cm}^{2}$ (from low to high current densities). The beneficial effects can be assigned to the ohmic losses and to the mass transport losses with a share of approximately two thirds and one third, respectively. The results of the $i / E$-curves are in line with the ones of the EIS-measurements, as discussed below. However, two questions now arise: (i) can the improvement be maintained, i.e. is the system stable? (ii) What are the underlying phenomena leading to the improvement. The first question will be answered in the following section by performing a short-term stress test.

Short-term stress test. To date, no accelerated stress tests for PEMWE cells are available, which have been validated and accepted by the community. ${ }^{5}$ Therefore, a specifically developed measurement protocol is used. As originally reported in ref. 27 , it includes repeating constant current density phases at $4 \mathrm{~A} \mathrm{~cm}^{-2}$, each lasting for approximately 30 hours. The HFR is furthermore measured every five minutes. As highlighted in Fig. 4a with "EC", in between these phases an electrochemical characterization 
phase with each three cycles of $i / E$-, EIS- and CI-measurements are performed. Thus, the durability of the cells is investigated at relatively high current densities respectively cell voltages for up to 150 hours (depending on the sample).

Similar to the results of the aforementioned overpotential analysis at the BOT, a significant and consistent reduction in the cell voltage of about $60 \mathrm{mV}$ is noticed for the L-PTLs, compared to the P-PTLs, during the $30 \mathrm{~h}$ periods at $4 \mathrm{~A} \mathrm{~cm}^{-2}$. Interestingly, this difference seems to be relatively stable across the individual $30 \mathrm{~h}$ cycles. However, irrespectively of the offset, the cell voltage curves of the pristine and the laser-structured PTL show qualitatively analogous behavior.
Within each constant current density phase, the cell voltage usually increases over time. However, when applying the electrochemical characterization phase, during which smaller current densities/cell voltages are applied, and switching back to the prior current density level, it can be observed that the cell voltage is reduced. This phenomenon is discussed hereinafter using the terms apparent degradation and real degradation. In literature, the terms reversible and irreversible are often used for this purpose. In this work, the real degradation is defined as the difference between the first measurement point of the next $30 \mathrm{~h}$ phase and the first measurement point of the previous $30 \mathrm{~h}$ phase. Thus, the real degradation rate quantifies the cell voltage
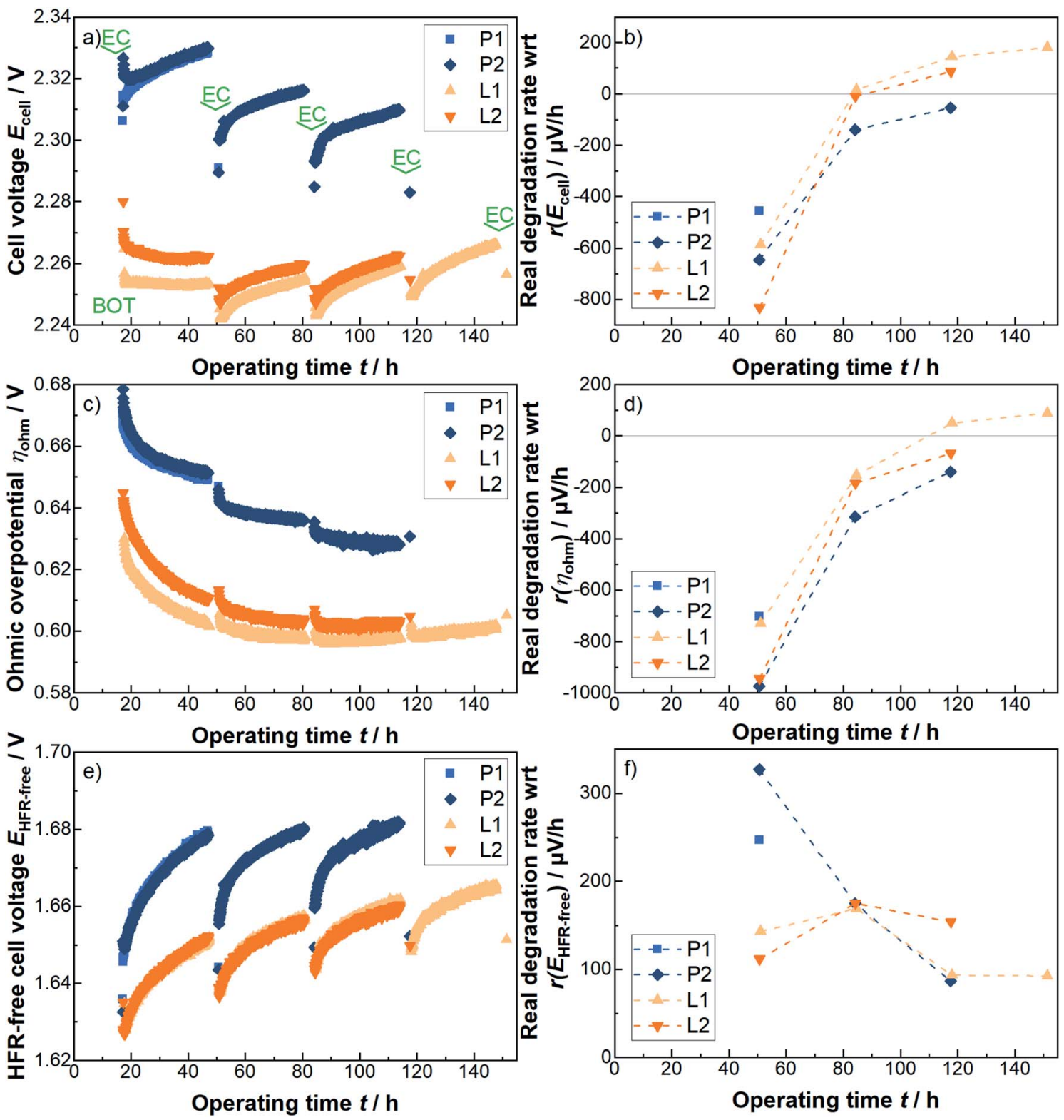

Fig. 4 Short-term stress test at $4 \mathrm{~A} \mathrm{~cm}^{-2}$ for several $30 \mathrm{~h}$ periods with respect to (a) the cell voltage, (c) the ohmic overpotential and (e) the HFRfree cell voltage. The corresponding real degradation rates are shown in (b), (d) and (f), respectively. The real degradation is the difference between the first measurement point of the next $30 \mathrm{~h}$ phase and the first measurement point of the previous $30 \mathrm{~h}$ phase, hereby positive values mean an actual worsening and negative values mean an actual improvement of e.g. the cell voltage (cf. text). In between the $30 \mathrm{~h}$ periods the cells are electrochemically characterized using i/E-curves and EIS-measurements, as indicated in (a) with "EC". The BOT is also highlighted in (a). 
losses that cannot be recovered without further effort. In contrast to the real degradation rate, the apparent degradation rate, adapted from PEM fuel cell literature (see e.g. ref. 33-35), is the difference between the last and the first measurement point at each constant current phase, normalized to the corresponding $30 \mathrm{~h}$ period, and corrected by the corresponding real degradation rate.

The rate of the real degradation with respect to the cell voltage is shown in Fig. 4b. It can be observed, that the real degradation rate with respect to the cell voltage is qualitatively and quantitatively similar to a large extent for all samples. It should be noted that here positive values represent an actual worsening of the cell voltage and accordingly negative values an actual improvement, since both degradation and so-called running-in processes take place concurrently. The difference between the cells with pristine and with laser-structured PTLs is increasingly diminishing in size. Whether this trend would continue and for how long is of great interest, but, unfortunately goes beyond the scope of this work. Quantitatively, the values are in line with previous results. ${ }^{27}$ In contrast, the apparent degradation rate of the laser-structured PTLs seem to be even smaller either due to the laser treatment or due to the simple fact that they operate at lower cell voltages.

To further elucidate the origin of the $60 \mathrm{mV}$ improvement, the individual overpotentials are analyzed in Fig. 4c-f. With respect to the ohmic overpotentials of each two cells with pristine and with laser-structured PTLs shown in Fig. 4c, a total improvement of about $40 \mathrm{mV}$ can be observed. Most of the degradation rate with respect to the cell voltage can be assigned to the ohmic region, as shown in Fig. $4 \mathrm{~b}$ and d. Consequently and similar to before, the remaining one third of the improvement is located in the HFR-free cell voltage, as shown in Fig. 4e. In contrast, the corresponding degradation rate with respect to the HFR-free cell voltage is in the positive value range at each measuring point for all cells.

To better understand the ongoing changes and to look more closely into the changes in the kinetic and mass transport region, the $i / E$-curves, including HFR-measurements and EISmeasurements from $100 \mathrm{kHz}$ to $100 \mathrm{mHz}$, are analyzed again. The corresponding points in time are of these individual electrochemical characterization phases and are highlighted with "EC" in Fig. 4a.

While hardly any differences can be seen in the kinetics during the short-term stress test, significant changes in the mass transport overpotential are observed, as shown in Fig. 5a in the form of HFR-free polarization curves together with the corresponding extrapolated Tafel-lines. It should be noted, that here only the results at the BOT and after the first $30 \mathrm{~h}$ (i.e. $47 \mathrm{~h}$ total testing time) constant current density period at $4 \mathrm{~A} \mathrm{~cm}^{-2}$ are compared, as unfortunately data is not available for all cells at later times. Furthermore, the HFR-free cell voltage difference between the two measurement points is provided for each sample separately in Fig. $5 \mathrm{~b}$. It can be highlighted, that the observed degradation shows a pseudo-linear behavior with the current density and a corresponding resistance increase of about 2-3 $\mathrm{m} \Omega \mathrm{cm}^{2}$ above about $2 \mathrm{~A} \mathrm{~cm}^{2}$. In comparison to the laser-structured PTLs a tendency towards slightly lower
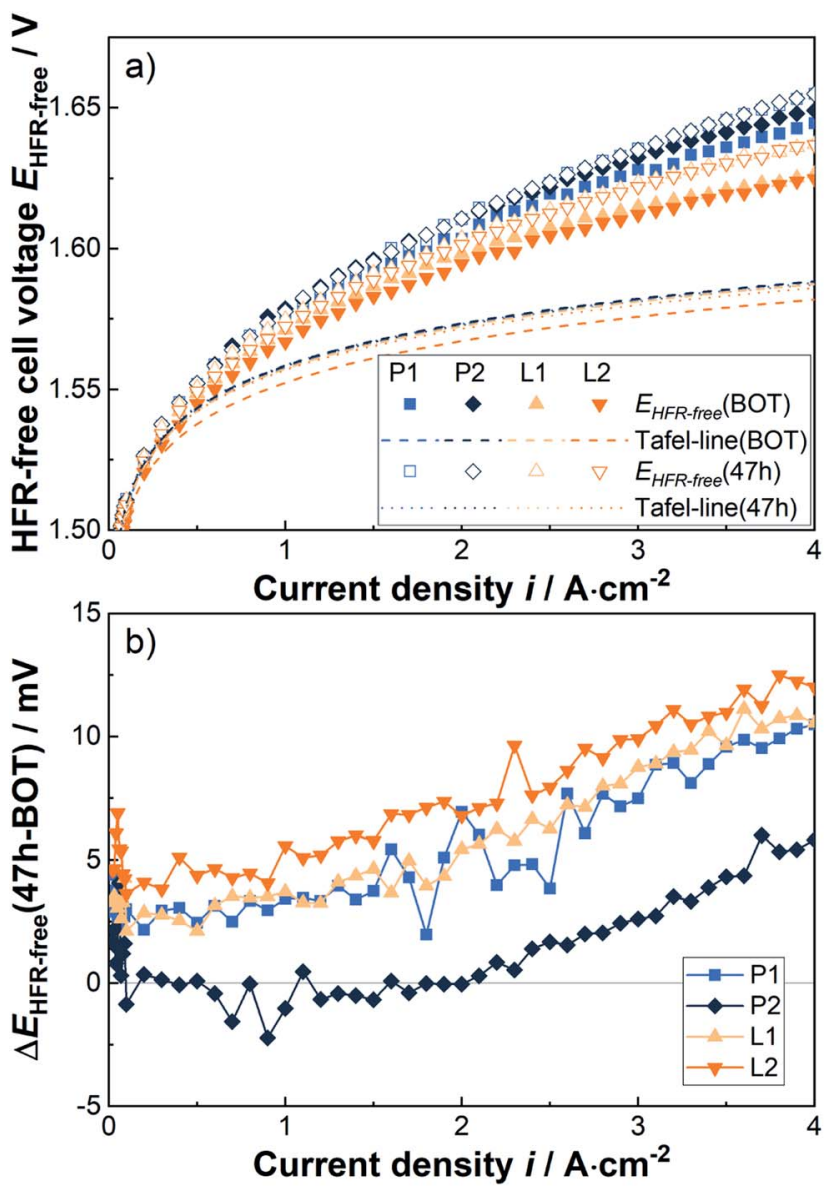

Fig. 5 (a) HFR-free cell voltage and extrapolated Tafel-lines at BOT and after the first $30 \mathrm{~h}$ period at $4 \mathrm{~A} \mathrm{~cm}^{-2}$, i.e. $47 \mathrm{~h}$, and (b) the corresponding difference in HFR-free cell voltage.

degradation rates is observed for the pristine PTLs. Thus, the improvements in the mass transport resistance due to the laserstructuring at relevant current densities are slightly reduced from about 5 to $4 \mathrm{~m} \Omega \mathrm{cm}^{2}$ from BOT to the $47 \mathrm{~h}$ benchmark.

The following paragraphs consider the changes across the entire stress test with the help of EIS-measurements. Therefore, the relatively well determinable HFR, the low frequency resistance (LFR) and the difference between both, i.e. the charge transfer resistance, are considered, as shown in Fig. 6a. A more in-depth analysis using e.g. electrical equivalent circuits is postponed due to different and partly contradictory interpretations of the capacitive effects, as discussed in ref. 36. In any case, the HFRs measured at representative current densities of $0.1,0.4,1$ and $4 \mathrm{~A} \mathrm{~cm}^{-2}$ are shown in Fig. $6 \mathrm{c}$ to f. In Fig. $6 \mathrm{~b}$ the difference between the LFR and the HFR, determined at the same current densities, is compared. The HFR is typically obtained at approximately $3 \mathrm{kHz}$, the LFR at approximately $1 \mathrm{~Hz}$. For illustration, also full spectra of EIS-measurements at $1 \mathrm{~A} \mathrm{~cm}^{-2}$ of the $\mathrm{P} 2$ and $\mathrm{L} 2$ cells at BOT and after $117 \mathrm{~h}$ are shown in the first quadrant of a Nyquist plot in Fig. 6 a.

The previously mentioned reduction in the HFR at the BOT of about 5-9 $\mathrm{m} \Omega \mathrm{cm}^{2}$ at technically relevant current densities is visible again. Later on this difference decreases slightly to e.g. 5- 

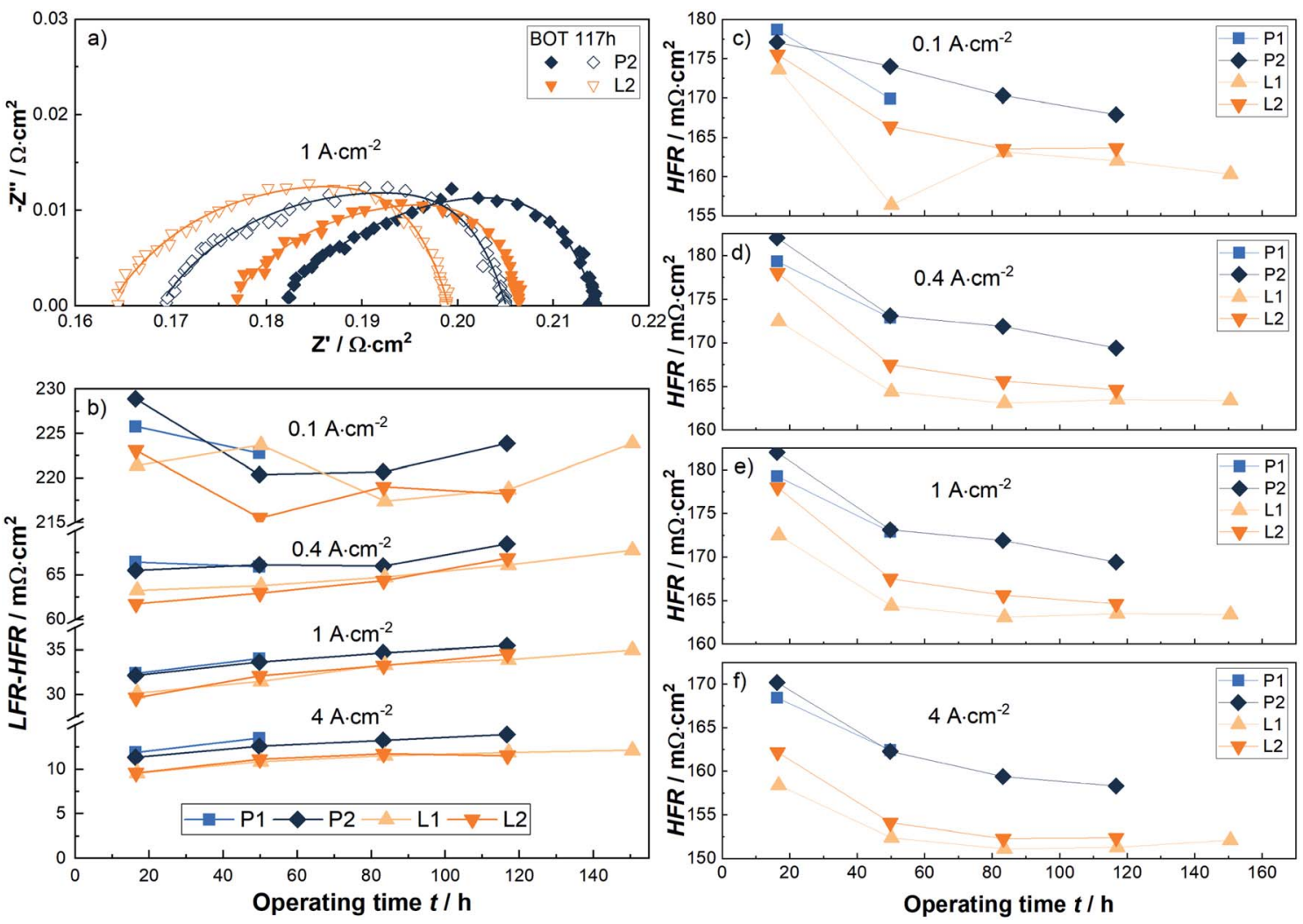

Fig. 6 (a) Nyquist plot shows exemplary EIS measurements for cells with the pristine P2-PTL and the laser-structured L2-PTL at $1 \mathrm{~A} \mathrm{~cm}^{-2}$. The HFR is usually obtained about at $3 \mathrm{kHz}$, the LFR at about $1 \mathrm{~Hz}$. Fits are mere guide-to-the-eye. (b) The difference of the LFR and the HFR, i.e. the charge transfer resistance, at indicated, representative current densities for all cells as a function of the operating time. In (c) to (f) the HFR measurements for all cells are shown at $0.1 \mathrm{~A} \mathrm{~cm}^{-2}, 0.4 \mathrm{~A} \mathrm{~cm}^{-2}, 1 \mathrm{~A} \mathrm{~cm}^{-2}$ and $4 \mathrm{~A} \mathrm{~cm}^{2}$, respectively.

$6 \mathrm{~m} \Omega \mathrm{cm}^{2}$. It can be highlighted, that the improvement of the HFR is relatively stable over the entire short-term stress test. In contrast, with respect to the charge transfer resistance (LFRHFR, Fig. 6b), a reduction of about $2 \mathrm{~m} \Omega \mathrm{cm}^{2}$ is obtained at relevant current densities and operating time.

In summary, the results of the initial overpotential analysis at the BOT can also be largely confirmed when applying a shortterm stress test of up to 150 hours at $4 \mathrm{~A} \mathrm{~cm}^{-2}$. The maintained improvements occur in the ohmic and mass transport resistances and amount to about 6 and $2 \mathrm{~m} \Omega \mathrm{cm}^{2}$ or three quarters to one quarter, respectively. Of course, a further investigation of the influence of the laser-structuring of the PTL surface on the durability over a practically relevant time frame of several months to years is of great interest, but unfortunately beyond the scope of this work. Instead, morphological and structural changes both of the PTL and CL surface at their interface are discussed in the next section to better understand the electrochemically measured differences.

\section{Physical characterization after cell tests}

Both the electrical ex situ tests and the electrochemical operando cell tests prove that the laser-structuring has beneficial influences towards the ohmic overpotential, namely the electrical interfacial contact resistances, and the mass transport overpotential. Keeping in mind the morphological changes due to the laser treatment of the PTL surface towards the CL ( $c f$. Fig. 1), it is of interest whether or not there are changes between before/after cell tests and different changes between the pristine and laser-structured titanium samples. Such changes are conceivable as a result of stresses of e.g. mechanical, chemical and electrochemical nature. Next to the PTL, the anodic CL is also investigated to complete the impression of their interface. For the physical characterization, the cells P2 and L2 are selected and serve as reference, since both were examined for the same duration of about $120 \mathrm{~h}$ in the short-term stress test.

SEM-images after cell tests are shown in Fig. 7. At the figure's top row, the pristine and at the bottom row the laser-structured PTLs are presented. With respect to the pristine PTLs, marked difference between the region underneath the ribs (shown here in Fig. 7 top) and the channels (shown in the supplemental material in Fig. S1 $\dagger$ ) is noticeable. Deposits of the CL are found almost exclusively underneath the ribs of the flow field in the form of tiny flakes in the nanometer range up to larger, ripped lump in the micrometer range. This can be explained due to a gradient of the contact pressure from the region underneath the ribs to the region underneath the channels ( $c f$. ref. 17). Thus, underneath the channels no significant change can be detected from before to after the cell tests. In contrast, for the laser-structured PTLs little to no deposits of the CL are to be found anywhere. Consequently, no difference is ascertainable 


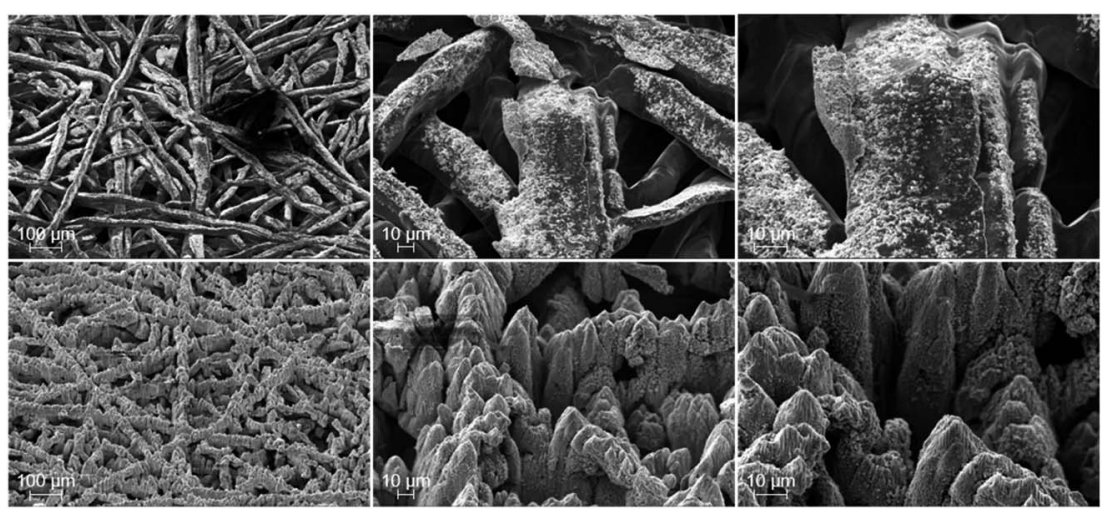

Fig. 7 SEM-images after PEMWE-cell tests of the PTL surface towards the CL underneath the rib for the pristine PTL (top) and laser-structured PTL (bottom) each at magnifications of $100 \times, 500 \times$ and $1000 \times$, from left to right. Deposits of the CL are seen as brightly shimmering flakes only in the pristine PTL images. These deposits are increasingly visible underneath the ribs of the flow field here (cf. SEM-images below the channels in Fig. S1 $\uparrow$ ), as the contact pressure is significantly higher. In contrast, little to no deposits of the CL can be identified for the laser-structured PTL. Consequently, no differences between the region below the ribs and channels of the flow field can be found. The SEM-images correspond to the aforementioned PEMWE-cell test results obtained with the samples P2 (pristine PTL) and L2 (laser-structured PTL).

between the region underneath the ribs and channels in this way (Fig. 7 top and S1 in the ESI†). It can be highlighted that only the fibers of the pristine PTLs underneath the ribs adhere to deposits of the CL. Consequently, the laser-structuring prevents that the catalyst layer is pulled apart when the cell is disassembled and may offer a better reusability of the PTLs. The underlying reason must be discovered in future work. On the other hand, compared to the SEM-images of the laserstructured PTLs before the electrochemical characterization, the substructure made of tiny flakes on the surface of the tips are largely gone and a lamellar like structure can be observed. The order of magnitude of this structure lies in the range of the wavelength of the laser and corresponds to an enhanced appearance of the LIPSS. ${ }^{28}$ With respect to the apparently vanished flakes, it can be hypothesized that they are dispersed due to the mechanical and/or electrochemical stress or that they are deposited into the catalyst layer. More importantly however, the laser-structured tips withstand the electrolysis conditions and appear stable.
Therefore, the question arises which morphological changes can be seen on the opposite side of the anodic CL? In Fig. 8, topview SEM-images of the anodic CL show an imprint of the pristine (top) and laser-structured (bottom) PTLs. In both cases, a distinct difference between the region underneath the ribs (Fig. 8) and the channels (Fig. S2 $\dagger$ ) of the flow field is noticed and can be directly explained again by the varying contact pressure. Consequently, the imprint of the PTLs on the CL is much more pronounced below the ribs. Thus, both small and considerably larger cracks up to the micrometer range occur next to the fiber imprints in the pore space of the PTL. This feature is caused due to mechanical stress as a result of a locally inhomogeneous contact pressure, as described in e.g. ref. 22 and 37.

When observing the differences between the imprint of the pristine to the laser-structured PTLs, the laser-structuring clearly results in a much more rough and jagged CL surface. At the fiber interfacial contact area, the imprints of the tips can be seen compared to the rather smooth, compressed imprint of
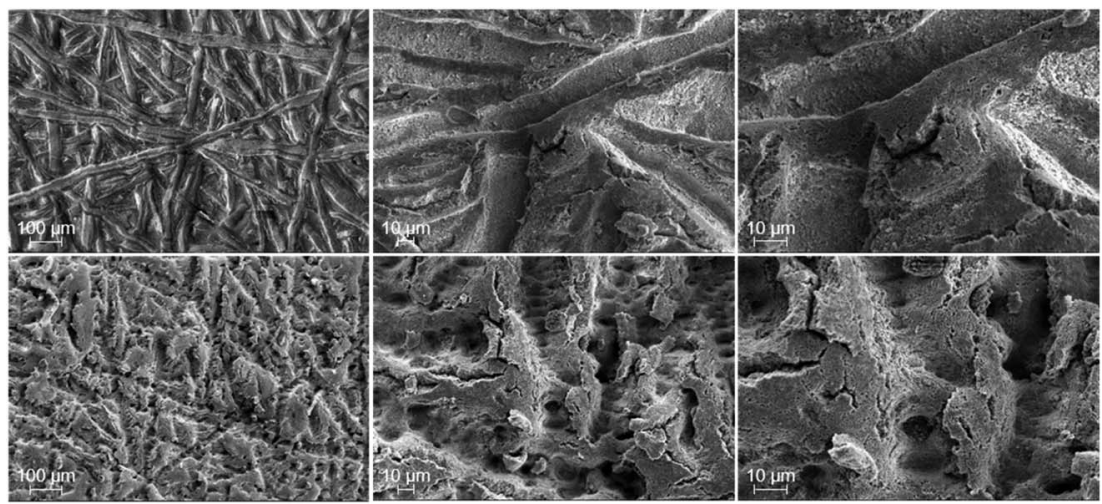

Fig. 8 SEM-images after PEMWE-cell tests of the anode side CCM surface underneath the rib of the cell with the pristine PTL (top) and with the laser-structured PTL (bottom) each at magnifications of $100 \times, 500 \times$ and $1000 \times$, from left to right. For both samples a distinct imprint of the PTL can be seen. This imprint is significantly less pronounced for the regions underneath the channels (cf. Fig. S2 $\dagger$ ). The SEM-images correspond to the aforementioned PEMWE-cell test results obtained with the samples P2 (pristine PTL) and L2 (laser-structured PTL). 
the untreated samples. Thus, the anodic CL surface morphology is not only cracked, serrated and expanded in the pore space of the fibers, but also at the interfacial contact area due to the laser-structuring.

In the next section, the electrical, electrochemical and physical results, which were predominantly considered individually, are gathered and discussed all together in order to better understand the observed influences of the laser treatment.

\section{Discussion}

Below the observed influences of the laser treatment are discussed systematically on the basis of the main overpotentials.

With respect to the OER kinetics, little to no influence is observed, as long as no change in the OER mechanism with increasing current density respectively overpotential is assumed, i.e. only one Tafel-slope exists. Following this very likely assumption, either the PTL/CL-interfacial contact area does not influence the kinetics at all or the influence of such a structuring in the sub-micrometer range is simply too small to be determined in this way. The latter could even be underlined by a relatively good performing material combination with a relatively over-engineered catalyst layer with a loading of about $2.5 \mathrm{mg} \mathrm{Ir} / \mathrm{cm}^{2}$ and with a thickness of about 10-15 $\mu \mathrm{m}$. Quite conceivable would be a change in the utilization of the catalyst layer as reported by Schuler et al. ${ }^{22}$ when drastically changing the fiber diameter and/or porosity of the PTL. This circumstance may play an even greater role once low-loading and thin catalyst layers are used, as the influence of the inplane electrical resistance of the CL rises in this case (see e.g. ref. 38).

In contrast, significant improvements are found in the ohmic and mass transport overpotential. The most likely and straightforward explanation of the reduced ohmic overpotential is due to an increase in the interfacial contact area between the anode PTL and CL. The area-specific HFR reduction of at least 6 $\mathrm{m} \Omega \mathrm{cm}^{2}$ has been verified qualitatively by $e x$ situ tests. A further reduction could also be achieved by using a protective coating of e.g. a thin iridium layer, as demonstrated by Liu et al. ${ }^{39}$ or by etching, as demonstrated by Bystron et al. ${ }^{\mathbf{4 0}}$ In correspondence with an increase in the specific interfacial contact area, a more homogenous local current density distribution seems conceivable. This might be measured on a slightly larger level using current density mapping devices (see e.g. ref. 41 and 42). Furthermore, it could be neither confirmed nor refuted, that the intrinsic electrical contact resistance of the PTL changes due to the laser-treatment. It can be hypothesized, however, that such a possible change is already invalidated by a rather stable HFR (improvement) over time during the aforementioned short-term stress test.

Much harder to interpret are the beneficial influences towards the mass transport overpotential with a reduction of at least $2 \mathrm{~m} \Omega \mathrm{cm}^{2}$, as here many different types of losses are merged. It would be conceivable that the much rougher and jagged CL surface could decrease the transport path length or improve the corresponding resistance of the supply of the liquid and gaseous water from the channel through the PTL bulk towards the active site of the catalyst and the removal of the dissolved oxygen through the ionomer phase and gaseous oxygen through the pores in the opposite direction. Since the gas crossover is apparently current density-dependent, ${ }^{43,44}$ complementary oxygen gas crossover measurements using non$\mathrm{H}_{2} / \mathrm{O}_{2}$-recombining cathodic catalyst could help to differentiate between the different origins. ${ }^{45}$ However, even if it is the case and these kind of mass transport resistances would be completely eliminated, which is of course unrealistic, these would amount to only a minor part of the observed voltage gain. Therefore, the remaining transport processes, which mainly take place in the pore space of the CL, PTL and in their interface, come into focus. Here the much rougher and jagged surface of the CL, both directly below the fibers and in the pore space in between, may promote the liquid water transport and/ or the gaseous oxygen and water transport. The proton transport resistance in the $\mathrm{CL}$, which is also part of the mass transport losses determined in this manner, can itself be determined via $\mathrm{H}_{2} / \mathrm{N}_{2}$-EIS-measurements considering a transmission line model (see e.g. ref. 18). Even if typical minor compromises must be made for PEMWE materials with regard to the humidification of the membrane and ionomer in the CL, Babic et al. have already stated that the proton transport resistance in the CL is influenced by the choice of PTL with changes in the single-digit $\mathrm{m} \Omega \mathrm{cm}^{2}$ range ${ }^{19}$ and by the catalyst loading in the CL respectively its morphology. ${ }^{20}$ Thus, it can be hypothesized, that changes in the mass transport overpotential originate in the proton transport resistance in the CL. Complementary experiments can confirm this. Of course, other losses in the mass transport overpotential that have not yet been taken into account can, of course, also be influenced by the laserstructuring.

Nevertheless, it can be summarized that both for the ohmic and mass transport losses, it is likely that several superimposed and possibly counteracting processes are influenced by the laser-structuring. More importantly, however, it turns out that the PTL/CL-interface, at least at the anode, plays an important and up until now mostly underrated role towards the performance and efficiency of an electrolyzer. Consequently, even more possibilities to further optimize the materials and components used are hereby demonstrated. In addition, further open questions still have to be addressed in ongoing work dealing with e.g. the long-term stability, optimum laserparameters and optimum contact pressure among others. Furthermore, the operating conditions should be extended according to the identified development goals towards even higher current densities, higher operating temperatures and possibly even higher (anode) pressures to handle the pressure depending apparent exchange current density and mass transport losses. ${ }^{46,47}$

\section{Conclusions}

The importance of the interface between the anode porous transport layer and the catalyst layer is underlined in this work. Since the titanium-based PTLs are preliminarily designed for 
needs in the filter industry, there is plenty of room for optimization for its application in the proton exchange membrane water electrolysis technology.

In this proof of concept paper, we have demonstrated that femtosecond laser-induced structuring of the fibers on the surface of the PTL, which is in contact with the catalyst layer, significantly improves the performance of the PEMWE cell. While little to no effects are assigned to the oxygen evolution reaction kinetics, beneficial effects are seen in the ohmic and mass transport overpotential. At the begin of test, the improvements can be assigned with a share of two thirds to the ohmic and one third to the mass transport overpotential. In the subsequent short-term stress test, this share changes slightly to three quarters to one quarter and remains stable at $4 \mathrm{~A} \mathrm{~cm}^{-2}$ during the 150 hours.

Thus, at the end of the test and over the entire current density range up to $4 \mathrm{~A} \mathrm{~cm}^{-2}$, a reduction in the equivalent area-specific cell resistance of at least $6 \mathrm{~m} \Omega \mathrm{cm}^{2}$ for the ohmic losses and of at least $2 \mathrm{~m} \Omega \mathrm{cm}^{2}$ for the mass transport losses are observed. Along with the physical characterization by means of scanning electron microscopy, the improvements in the high frequency resistance are assigned to reduced electrical interfacial contact resistances due to the increase in the specific surface area of the PTL fibers contacting the CL. Whether other aspects, such as the (local) current density and contact pressure distribution, are affected remains to be investigated in follow-up work.

In contrast, it appears that the beneficial effects in the mass transport domain have been rather difficult to explain so far. On the basis of the existing data and of the exclusion principle, the liquid water transport to and the gaseous oxygen transport from the active sites of the iridium-based catalysts seem responsible for it. This hypothesis is particularly underlined with top view SEM images of the CL surface after cell tests, showing a much rougher and jagged morphology, both below the fibers and below the pore space in between the fibers of the PTLs compared to the otherwise equivalent samples, which were in contact with the pristine (or unmodified) PTLs. For a better understanding, complementary experiments, e.g. investigating the influence towards the proton transport resistance in the CL and towards the gas crossover, are planned.

In addition, to achieve a better fundamental understanding of the origin of the improvements, the long-term stability over weeks to months is also of technical interest. Just as important is the systematic investigation of the probably materialdepending optimal laser-parameters. In closing, further insight into the importance of the interface between catalyst layer and porous transport layer could be obtained. It could be confirmed that it is possible to further improve the efficiency of the PEM water electrolyzer by further reducing the e.g. electrical interfacial contact resistance. With this in mind, a new possibility has been presented, which can be of great technical relevance for material developers and stack designers in particular.

\section{Conflicts of interest}

There are no conflicts to declare.

\section{Acknowledgements}

The authors gratefully acknowledge the Energy Research Centre of Lower Saxony (EFZN) for the establishment of a discussion platform within the framework of the Competence Network Water Electrolysis of Lower Saxony, from which this cooperation emerged. We also thank Alexander Bomm and Patrick Trinke for their technical and scientific support in the preliminary work, as well as Inga Beyers for proofreading. The publication of this article was funded by the Open Access fund of Leibniz Universität Hannover.

\section{References}

1 C. J. Barnhart, M. Dale, A. R. Brandt and S. M. Benson, The energetic implications of curtailing versus storing solarand wind-generated electricity, Energy Environ. Sci., 2013, 6, 2804, DOI: 10.1039/c3ee41973h.

2 A. Buttler and H. Spliethoff, Current status of water electrolysis for energy storage, grid balancing and sector coupling via power-to-gas and power-to-liquids: A review, Renewable Sustainable Energy Rev., 2018, 82, 2440-2454, DOI: 10.1016/j.rser.2017.09.003.

3 M. Kopp, D. Coleman, C. Stiller, K. Scheffer, J. Aichinger, B. Scheppat and E. Mainz, Technical and economic analysis of the worldwide largest Power-to-Gas plant with PEM electrolysis, Int. J. Hydrogen Energy, 2017, 42, 1331113320, DOI: 10.1016/j.ijhydene.2016.12.145.

4 K. Ayers, N. Danilovic, R. Ouimet, M. Carmo, B. Pivovar and M. Bornstein, Perspectives on Low-Temperature Electrolysis and Potential for Renewable Hydrogen at Scale, Annual review of chemical and biomolecular engineering, 2019, 10, 219-239, DOI: 10.1146/annurev-chembioeng-060718-030241.

5 U. Babic, M. Suermann, F. N. Büchi, L. Gubler and T. J. Schmidt, Critical Review-Identifying Critical Gaps for Polymer Electrolyte Water Electrolysis Development, J. Electrochem. Soc., 2017, 164, F387-F399, DOI: 10.1149/ 2.1441704jes.

6 M. Carmo, D. L. Fritz, J. Mergel and D. Stolten, A comprehensive review on PEM water electrolysis, Int. J. Hydrogen Energy, 2013, 38, 4901-4934, DOI: 10.1016/ j.ijhydene.2013.01.151.

7 S. A. Grigoriev, P. Millet, S. A. Volobuev and V. N. Fateev, Optimization of porous current collectors for PEM water electrolysers, Int. J. Hydrogen Energy, 2009, 34, 4968-4973, DOI: 10.1016/j.ijhydene.2008.11.056.

8 H. Ito, T. Maeda, A. Nakano, C. M. Hwang, M. Ishida, A. Kato and T. Yoshida, Experimental study on porous current collectors of PEM electrolyzers, Int. J. Hydrogen Energy, 2012, 37, 7418-7428, DOI: 10.1016/j.ijhydene.2012.01.095.

9 H. Ito, T. Maeda, A. Nakano, A. Kato and T. Yoshida, Influence of pore structural properties of current collectors on the performance of proton exchange membrane electrolyzer, Electrochim. Acta, 2013, 100, 242-248, DOI: 10.1016/j.electacta.2012.05.068.

10 C. M. Hwang, M. Ishida, H. Ito, T. Maeda, A. Nakano, A. Kato and T. Yoshida, Effect of titanium powder loading in gas 
diffusion layer of a polymer electrolyte unitized reversible fuel cell, J. Power Sources, 2012, 202, 108-113, DOI: 10.1016/j.jpowsour.2011.11.041.

11 Z. Kang, J. Mo, G. Yang, Y. Li, D. A. Talley, S. T. Retterer, D. A. Cullen, T. J. Toops, M. P. Brady, G. Bender, B. S. Pivovar, J. B. Green and F.-Y. Zhang, Thin film surface modifications of thin/tunable liquid/gas diffusion layers for high-efficiency proton exchange membrane electrolyzer cells, Appl. Energy, 2017, 206, 983-990, DOI: 10.1016/ j.apenergy.2017.09.004.

12 Z. Kang, J. Mo, G. Yang, Y. Li, D. A. Talley, B. Han and F.-Y. Zhang, Performance Modeling and Current Mapping of Proton Exchange Membrane Electrolyzer Cells with Novel Thin/Tunable Liquid/Gas Diffusion Layers, Electrochim. Acta, 2017, 255, 405-416, DOI: 10.1016/ j.electacta.2017.09.170.

13 Z. Kang, J. Mo, G. Yang, S. T. Retterer, D. A. Cullen, T. J. Toops, J. B. Green Jr, M. M. Mench and F.-Y. Zhang, Investigation of thin/well-tunable liquid/gas diffusion layers exhibiting superior multifunctional performance in low-temperature electrolytic water splitting, Energy Environ. Sci., 2017, 10, 166-175, DOI: 10.1039/c6ee02368a.

14 Z. Kang, S. Yu, G. Yang, Y. Li, G. Bender, B. S. Pivovar, J. B. Green and F.-Y. Zhang, Performance improvement of proton exchange membrane electrolyzer cells by introducing in-plane transport enhancement layers, Electrochim. Acta, 2019, 316, 43-51, DOI: 10.1016/ j.electacta.2019.05.096.

15 M. Suermann, T. J. Schmidt and F. N. Büchi, Cell Performance Determining Parameters in High Pressure Water Electrolysis, Electrochim. Acta, 2016, 211, 989-997, DOI: 10.1016/j.electacta.2016.06.120.

16 M. Suermann, K. Takanohashi, A. Lamibrac, T. J. Schmidt and F. N. Büchi, Influence of Operating Conditions and Material Properties on the Mass Transport Losses of Polymer Electrolyte Water Electrolysis, J. Electrochem. Soc., 2017, 164, F973-F980, DOI: 10.1149/2.13517109jes.

17 M. Bernt and H. A. Gasteiger, Influence of Ionomer Content in IrO 2/TiO 2 Electrodes on PEM Water Electrolyzer Performance, J. Electrochem. Soc., 2016, 163, F3179-F3189, DOI: $10.1149 / 2.0231611$ jes.

18 K. C. Neyerlin, W. Gu, J. Jorne, A. Clark and H. A. Gasteiger, Cathode Catalyst Utilization for the ORR in a PEMFC, Electrochim. Acta, 2007, 154, B279, DOI: 10.1149/1.2400626.

19 U. Babic, T. J. Schmidt and L. Gubler, CommunicationContribution of Catalyst Layer Proton Transport Resistance to Voltage Loss in Polymer Electrolyte Water Electrolyzers, J. Electrochem. Soc., 2018, 165, J3016-J3018, DOI: 10.1149/ 2.0031815jes.

20 U. Babic, E. Nilsson, A. Pătru, T. J. Schmidt and L. Gubler, Proton Transport in Catalyst Layers of a Polymer Electrolyte Water Electrolyzer: Effect of the Anode Catalyst Loading, J. Electrochem. Soc., 2019, 166, F214-F220, DOI: 10.1149/2.0341904jes.

21 T. Schuler, R. de Bruycker, T. J. Schmidt and F. N. Büchi, Polymer Electrolyte Water Electrolysis: Correlating Porous Transport Layer Structural Properties and Performance:
Part I. Tomographic Analysis of Morphology and Topology, J. Electrochem. Soc., 2019, 166, F270-F281, DOI: 10.1149/ 2.0561904jes.

22 T. Schuler, T. J. Schmidt and F. N. Büchi, Polymer Electrolyte Water Electrolysis: Correlating Performance and Porous Transport Layer Structure: Part II. Electrochemical Performance Analysis, J. Electrochem. Soc., 2019, 166, F555F565, DOI: 10.1149/2.1241908jes.

23 M. Koj, T. Gimpel, W. Schade and T. Turek, Laser structured nickel-iron electrodes for oxygen evolution in alkaline water electrolysis, Int. J. Hydrogen Energy, 2019, 44, 12671-12684, DOI: 10.1016/j.ijhydene.2019.01.030.

24 A. Gabler, C. I. Müller, T. Rauscher, T. Gimpel, R. Hahn, M. Köhring, B. Kieback, L. Röntzsch and W. Schade, Ultrashort-pulse laser structured titanium surfaces with sputter-coated platinum catalyst as hydrogen evolution electrodes for alkaline water electrolysis, Int. J. Hydrogen Energy, 2018, 43, 7216-7226, DOI: 10.1016/ j.ijhydene.2018.02.130.

25 T. Rauscher, C. I. Müller, A. Gabler, T. Gimpel, M. Köhring, B. Kieback, W. Schade and L. Röntzsch, Femtosecond-laser structuring of $\mathrm{Ni}$ electrodes for highly active hydrogen evolution, Electrochim. Acta, 2017, 247, 1130-1139, DOI: 10.1016/j.electacta.2017.07.074.

26 G. Alberti, R. Narducci and M. Sganappa, Effects of hydrothermal/thermal treatments on the water-uptake of Nafion membranes and relations with changes of conformation, counter-elastic force and tensile modulus of the matrix, J. Power Sources, 2008, 178, 575-583, DOI: 10.1016/j.jpowsour.2007.09.034.

27 M. Suermann, B. Bensmann and R. Hanke-Rauschenbach, Degradation of Proton Exchange Membrane (PEM) Water Electrolysis Cells: Looking Beyond the Cell Voltage Increase, J. Electrochem. Soc., 2019, 166, F645-F652, DOI: 10.1149/2.1451910jes.

28 S. Höhm, M. Herzlieb, A. Rosenfeld, J. Krüger and J. Bonse, Dynamics of the formation of laser-induced periodic surface structures (LIPSS) upon femtosecond two-color double-pulse irradiation of metals, semiconductors, and dielectrics, Appl. Surf. Sci., 2016, 374, 331-338, DOI: 10.1016/ j.apsusc.2015.12.129.

29 C. Rozain and P. Millet, Electrochemical characterization of Polymer Electrolyte Membrane Water Electrolysis Cells, Electrochim. Acta, 2014, 131, 160-167, DOI: 10.1016/ j.electacta.2014.01.099.

30 J. O. '. M. Bockris, A. K. N. Reddy and M. E. Gamboa-Aldeco, Modern Electrochemistry 2A, Kluwer Academic Publishers, Boston, 2002.

31 J. Durst, A. Siebel, C. Simon, F. Hasché, J. Herranz and H. A. Gasteiger, New insights into the electrochemical hydrogen oxidation and evolution reaction mechanism, Energy Environ. Sci., 2014, 7, 2255-2260, DOI: 10.1039/ c4ee00440j.

32 A. R. Kucernak and C. Zalitis, General Models for the Electrochemical Hydrogen Oxidation and Hydrogen Evolution Reactions: Theoretical Derivation and Experimental Results under Near Mass-Transport Free 
Conditions, J. Phys. Chem. C, 2016, 120, 10721-10745, DOI: 10.1021/acs.jpcc.6b00011.

33 S. Kundu, M. Fowler, L. C. Simon and R. Abouatallah, Reversible and irreversible degradation in fuel cells during Open Circuit Voltage durability testing, J. Power Sources, 2008, 182, 254-258, DOI: 10.1016/j.jpowsour.2008.04.009.

34 P. Gazdzick, J. Mitzel, D. Garcia Sanchez, M. Schulze and K. A. Friedrich, Evaluation of reversible and irreversible degradation rates of polymer electrolyte membrane fuel cells tested in automotive conditions, J. Power Sources, 2016, 327, 86-95, DOI: 10.1016/j.jpowsour.2016.07.049.

35 G. Tsotridis, A. Pilenga, G. de Marco and T. Malkow, EU harmonised test protocols for PEMFC MEA testing in single cell configuration for automotive applications, Publications Office, Luxembourg, 2015.

36 M. Suermann, A. Pătru, T. J. Schmidt and F. N. Büchi, High pressure polymer electrolyte water electrolysis: Test bench development and electrochemical analysis, Int. J. Hydrogen Energy, 2017, 42, 12076-12086, DOI: 10.1016/ j.ijhydene.2017.01.224.

37 P. Millet, N. Mbemba, S. A. Grigoriev, V. N. Fateev, A. Aukauloo and C. Etiévant, Electrochemical performances of PEM water electrolysis cells and perspectives, Int. J. Hydrogen Energy, 2011, 36, 4134-4142, DOI: 10.1016/j.ijhydene.2010.06.105.

38 M. Bernt, A. Siebel and H. A. Gasteiger, Analysis of Voltage Losses in PEM Water Electrolyzers with Low Platinum Group Metal Loadings, J. Electrochem. Soc., 2018, 165, F305-F314, DOI: 10.1149/2.0641805jes.

39 C. Liu, M. Carmo, G. Bender, A. Everwand, T. Lickert, J. L. Young, T. Smolinka, D. Stolten and W. Lehnert, Performance enhancement of PEM electrolyzers through iridium-coated titanium porous transport layers, Electrochem. Commun., 2018, 97, 96-99, DOI: 10.1016/ j.elecom.2018.10.021.

40 T. Bystron, M. Vesely, M. Paidar, G. Papakonstantinou, K. Sundmacher, B. Bensmann, R. Hanke-Rauschenbach and K. Bouzek, Enhancing PEM water electrolysis efficiency by reducing the extent of Ti gas diffusion layer passivation, J. Appl. Electrochem., 2018, 48, 713-723, DOI: 10.1007/s10800-018-1174-6.

41 B. Verdin, F. Fouda-Onana, S. Germe, G. Serre, P. A. Jacques and P. Millet, Operando current mapping on PEM water electrolysis cells. Influence of mechanical stress, Int. J. Hydrogen Energy, 2017, 42, 25848-25859, DOI: 10.1016/ j.ijhydene.2017.08.189.

42 C. Immerz, B. Bensmann, P. Trinke, M. Suermann and R. Hanke-Rauschenbach, Local Current Density and Electrochemical Impedance Measurements within $50 \mathrm{~cm}$ Single-Channel PEM Electrolysis Cell, J. Electrochem. Soc., 2018, 165, F1292-F1299, DOI: 10.1149/2.0411816jes.

$43 \mathrm{M}$. Schalenbach and D. Stolten, High-pressure water electrolysis: Electrochemical mitigation of product gas crossover, Electrochim. Acta, 2015, 156, 321-327, DOI: 10.1016/j.electacta.2015.01.010.

44 P. Trinke, G. P. Keeley, M. Carmo, B. Bensmann and R. Hanke-Rauschenbach, Elucidating the Effect of Mass Transport Resistances on Hydrogen Crossover and Cell Performance in PEM Water Electrolyzers by Varying the Cathode Ionomer Content, J. Electrochem. Soc., 2019, 166, F465-F471, DOI: 10.1149/2.0171908jes.

45 P. Trinke, B. Bensmann and R. Hanke-Rauschenbach, Experimental evidence of increasing oxygen crossover with increasing current density during PEM water electrolysis, Electrochem. Commun., 2017, 82, 98-102, DOI: 10.1016/ j.elecom.2017.07.018.

46 S. A. Grigor'ev, M. M. Khaliullin, N. V. Kuleshov and V. N. Fateev, Electrolysis of Water in a System with a Solid Polymer Electrolyte at Elevated Pressure, Russ. J. Electrochem., 2001, 37, 819-822, DOI: 10.1023/ A:1016735003101.

47 M. Suermann, T. Kiupel, T. J. Schmidt and F. N. Büchi, Electrochemical Hydrogen Compression: Efficient Pressurization Concept Derived from an Energetic Evaluation, J. Electrochem. Soc., 2017, 164, F1187-F1195, DOI: $10.1149 / 2.1361712$ jes. 